\title{
NIP FOR SOME PAIR-LIKE THEORIES
}

\author{
GARETH BOXALL
}

\begin{abstract}
Generalising work from [2] and [6], we give sufficient conditions for a theory $T_{P}$ to inherit $N I P$ from $T$, where $T_{P}$ is an expansion of the theory $T$ by a unary predicate $P$. We apply our result to theories, studied in [1], of the real field with a subgroup of the unit circle.
\end{abstract}

\section{INTRODUCTION}

We consider the situation where $T$ is a complete one-sorted theory with infinite models, $L$ is the language of $T, P$ is a new unary predicate, $L_{P}=$ $L \cup\{P\}, M \models T$ and $T_{P}$ is the complete theory of some expansion of $M$ to $L_{P}$. Our main result provides sufficient conditions for $T_{P}$ to inherit NIP from $T$. It is a common generalisation of two other recent results, one of Berenstein, Dolich and Onshuus in [2] and one of Günaydın and Hieronymi in [6]. With respect to the result from [2], our generalisation removes the assumption that $P(M)$ be algebraically closed. With respect to the result from [6], it has the advantage that it works outside the setting where $T$ is o-minimal. We apply our result to theories of the real field with a predicate for a subgroup of the unit circle which were studied by Belegradek and Zilber in [1]. Similar theories were studied by van den Dries and Günaydın in [5] and by Berenstein, Ealy and Günaydın in [3] and shown to have NIP in [6].

This short paper has evolved over a period of time and benefited from many useful conversations, comments and corrections for which I thank Hans Adler, John Baldwin, Oleg Belegradek, Alexander Berenstein, Dugald Macpherson, Boris Zilber and the referee. Above all I would like to thank my PhD supervisor Anand Pillay for much generous assistance with this material, including suggesting the main approach used.

\section{A general Result}

First order logic is used throughout. The expansion of $M$ to $L_{P}$ is written as $(M, P(M))$. We work in $L_{P}$ (or $T_{P}$ ) except where we specifically indicate $L$ (or $T$ ). For example, acl denotes algebraic closure in the sense of $T_{P}$ while $a c l_{L}$ denotes algebraic closure in the sense of $T$. Similarly $t p(\bar{a} / B)$ is a complete type in the sense of $T_{P}$ while $t p_{L}(\bar{a} / B)$ is a complete type in the 
sense of $T$. Otherwise our notation is fairly standard. We abbreviate $A \cup B$ to $A B$ and sometimes sets are treated as tuples or vice versa. For a tuple of variables $\bar{x}=x_{1} \ldots x_{n}$ we abbreviate $P\left(x_{1}\right) \wedge \ldots \wedge P\left(x_{n}\right)$ to $P(\bar{x})$. Our main result is the following.

Theorem 2.1. $T_{P}$ has NIP if, for any $(M, P(M)) \prec(N, P(N)) \prec(\bar{M}, P(\bar{M})) \models$ $T_{P}$ such that all three models are sufficiently saturated, the following conditions are satisfied:

(i) acl $_{L}$ is a pregeometry on $\bar{M}$,

(ii) if $b \in \bar{M} \backslash \operatorname{acl}_{L}(N P(\bar{M}))$ then $t p(b / N)$ is implied by $t p_{L}(b / N)$ in conjunction with the information that $b \notin a c l_{L}(N P(\bar{M}))$,

(iii) for every finite $n \geq 1$ and $\bar{f} \in P(\bar{M})^{n}$, there exist $\kappa \leq|M|$ and $\widehat{f} \in$ $P(\bar{M})^{\kappa}$ such that $\bar{f} \subseteq \widehat{f}$ and, for every $\widehat{f}^{\prime}=t p(\widehat{f} / M), \operatorname{tp}\left(\widehat{f}^{\prime} / N\right)$ is implied by $t p_{L}\left(\widehat{f}^{\prime} / N\right)$ in conjunction with $\operatorname{tp}\left(\widehat{f}^{\prime} / M\right)$,

(iv) $T$ has NIP.

The thinking behind condition (iii) should become clear in the light of Sections 3 and 4. However it would probably be helpful at this stage to mention a neater version of it which is sufficient for some interesting applications:

(iii) $^{\prime}$ for every finite $n \geq 1$ and $\bar{f} \in P(\bar{M})^{n}, t p(\bar{f} / N)$ is implied by $t p_{L}(\bar{f} / N)$ in conjunction with the information that $\bar{f} \in P(\bar{M})^{n}$.

Clearly when (iii) ${ }^{\prime}$ replaces (iii) in the assumptions of Theorem 2.1, these assumptions are if anything strengthened. We shall comment further on (iii) ${ }^{\prime}$ in Section 3.

The independence property (the negation of NIP) was introduced by Shelah in [8]. Our proof of Theorem 2.1 uses the following fact due to a combination of Shelah and Poizat. Details are given in chapter 12 of [7].

Fact 2.2. The following conditions are equivalent and $T$ has NIP if and only if they are true:

(1) for every $M \prec N \models T$ such that both models are sufficiently saturated, there are no more than $2^{|M|}$ complete one-types $q(x)$ over $N$ such that $q(x)$ is finitely realisable in $M$,

(2) for every finite $n \geq 1$ and every $M \prec N \models T$ such that both models are sufficiently saturated, there are no more than $2^{|M|}$ complete $n$-types $q(\bar{x})$ over $N$ such that $q(\bar{x})$ is finitely realisable in $M$.

We conclude this section with a proof of Theorem 2.1. Let $(M, P(M)) \prec$ $(N, P(N)) \prec(\bar{M}, P(\bar{M})) \models T_{P}$ be such that all three models are sufficiently 
saturated. Let $b \in \bar{M}$ and suppose $t p(b / N)$ is finitely realisable in $M$. We show that there are no more than $2^{|M|}$ choices for $t p(b / N)$.

Case 1: Suppose $b \notin a c l_{L}(N P(\bar{M}))$. By condition (ii), $t p(b / N)$ is implied by $\operatorname{tp}_{L}(b / N)$ in conjunction with the information that $b \notin a c l_{L}(N P(\bar{M}))$. Clearly $t p_{L}(b / N)$ is finitely realisable in $M$. By condition (iv) and Fact $2.2(1)$, there are no more than $2^{|M|}$ choices for $t p_{L}(b / N)$. Therefore there are, in this case, no more than $2^{|M|}$ choices for $t p(b / N)$.

Case 2: Suppose $b \in \operatorname{acl}_{L}(N P(\bar{M}))$. Let $\bar{a} \bar{c} \bar{f}$ be a tuple such that $\bar{a} \in M^{k}$, $\bar{c} \in N^{l}$ and $\bar{f} \in P(\bar{M})^{n}$, for some $k, l, n<\omega$, and $b \in a c l_{L}(\bar{a} \bar{c} \bar{f})$. We may assume $\bar{c}$ is of minimal length. Suppose $\bar{c}$ is not empty (that is to say $l \neq 0$ ). It follows by condition (i) that $\bar{c}$ is not $a c l_{L}$-independent over $\bar{a} b \bar{f}$. Let $\varphi(\bar{x}, y, \bar{z}, \bar{w})$ be an $L$-formula which is realised by $\bar{a}, b, \bar{c}, \bar{f}$ and which witnesses the fact that $\bar{c}$ is not $a c l_{L}$-independent over $\bar{a} b \bar{f}$. Since $t p(b / \bar{a} \bar{c})$ is finitely realisable in $M$, the formula $\psi(\bar{a}, y, \bar{c}) \equiv(\exists \bar{w})[P(\bar{w}) \wedge \varphi(\bar{a}, y, \bar{c}, \bar{w})]$ is realisable in $M$ and so $\bar{c}$ is not $a c l_{L}$-independent over $M P(\bar{M})$. This contradicts the minimality of the length of $\bar{c}$. Therefore $\bar{c}$ is empty. Therefore $b \in \operatorname{acl}_{L}(M \bar{f})$.

Let $\kappa$ and $\widehat{f}$ be as in condition (iii). Let $p(y \widehat{w})=r(y) \wedge s(y \widehat{w})$ where $r(y)=t p(b / N)$ and $s(y \widehat{w})=t p(b \widehat{f} / M)$. Then $p(y \widehat{w})$ is finitely realisable in $M$. By a well known argument (you extend a filter-base to an ultrafilter and extract what you want from that), $p(y \widehat{w})$ extends to a complete type $p^{\prime}(y \widehat{w})$ over $N$ which is finitely realisable in $M$. Let $b^{\prime} \widehat{f}^{\prime}=p^{\prime}(y \widehat{w})$. Then $t p\left(b^{\prime} / N\right)=t p(b / N), t p\left(\widehat{f}^{\prime} / M\right)=t p(\widehat{f} / M)$ and $b^{\prime} \in \operatorname{acl}_{L}\left(M \widehat{f}^{\prime}\right)$.

By condition (iii), $t p\left(\widehat{f}^{\prime} / N\right)$ is implied by $t p_{L}\left(\widehat{f}^{\prime} / N\right)$ in conjunction with

$\operatorname{tp}\left(\widehat{f}^{\prime} / M\right)$. Clearly $t p_{L}\left(\widehat{f}^{\prime} / N\right)$ is finitely realisable in $M$. By condition (iv) and Fact 2.2(2), there are no more than $\left(2^{|M|}\right)^{|M|}$ choices for $t p_{L}\left(\widehat{f}^{\prime} / N\right)$. Therefore there are no more than $\left(2^{|M|}\right)^{|M|} \times\left(2^{|M|}\right)^{|M|}$ choices for $\operatorname{tp}\left(\widehat{f}^{\prime} / N\right)$. Therefore there are, in this case, no more than $\left(2^{|M|}\right)^{|M|} \times\left(2^{|M|}\right)^{|M|} \times|M| \times$ $|M|=2^{|M|}$ choices for $t p(b / N)$.

Adding the two cases together, there are no more than $2^{|M|}$ choices for $t p(b / N)$. Therefore $T_{P}$ has NIP, by Fact 2.2(1).

\section{Comparison with Results in [2] And [6]}

Theorem 2.7 of [2] makes use of the notion of $P$-independence which is defined as follows and makes sense provided $a c l_{L}$ is a pregeometry.

Definition 3.1. A set $A \subseteq M$ is said to be $P$-independent if $A$ is $a c l_{L^{-}}$ independent from $P(M)$ over $A \cap P(M)$.

We shall also want to speak of $P-t p(\bar{a})$ by which we mean the information that tells us which members of the tuple $\bar{a}$ belong to $P(M)$. The following is Theorem 2.7 from [2]. 
Theorem 3.2. $T_{P}$ has NIP if, for all $(M, P(M)) \models T_{P}$, the following conditions are satisfied:

(a) acl $_{L}$ is a pregeometry on $M$,

(b) for every finite $n \geq 1$, if $\bar{a}, \bar{b} \in M^{n}$ are such that both $\bar{a}$ and $\bar{b}$ are $P$-independent then $t p(\bar{a})=t p(\bar{b})$ provided both $t p_{L}(\bar{a})=t p_{L}(\bar{b})$ and $P$ $t p(\bar{a})=P-t p(\bar{b})$,

(c) $\operatorname{acl}_{L}(P(M))=P(M)$,

(d) $T$ has NIP.

The following result establishes that Theorem 2.1 is a generalisation of Theorem 3.2. The proof is a standard argument in this area. We include it for the sake of completeness. Note that when we speak of $P$-independence we mean with respect to the larger model $(\bar{M}, P(\bar{M}))$.

Proposition 3.3. The assumptions of Theorem 3.2 excluding (c) imply the assumptions of Theorem 2.1 even when (iii)' replaces (iii).

Proof. Suppose the assumptions of Theorem 3.2 are satisfied with the possible exception of (c). Let $(M, P(M)) \prec(N, P(N)) \prec(\bar{M}, P(\bar{M})) \models T_{P}$ be such that all three models are sufficiently saturated. Conditions (i) and (iv) follow immediately. Since $(N, P(N)) \prec(\bar{M}, P(\bar{M}))$ it is clear that, for every finite tuple $\bar{a}$ from $N$, there is a finite tuple $\bar{g}$ from $P(N)$ such that $\bar{a} \bar{g}$ is $P$-independent. Let $b \in \bar{M} \backslash \operatorname{acl}_{L}(N P(\bar{M}))$. For every $P$-independent tuple $\bar{a}$ from $N$, it is clear that $b \bar{a}$ is also $P$-independent. It follows by condition (b) that $t p(b / N)$ is implied by $t p_{L}(b / N)$ in conjunction with the information that $b \notin a c l_{L}(N P(\bar{M}))$. So condition (ii) is satisfied. Let $n \geq 1$ be finite and $\bar{f} \in P(\bar{M})^{n}$. For every $P$-independent tuple $\bar{a}$ from $N$ it is clear that $\bar{f} \bar{a}$ is also $P$-independent. It follows by condition (b) that $\operatorname{tp}(\bar{f} / N)$ is implied by $t p_{L}(\bar{f} / N)$ in conjunction with the information that $\bar{f} \in P(\bar{M})^{n}$. So condition (iii)' is satisfied.

Theorem 3.2 is used in [2] to show that if $T$ has NIP and is a geometric theory and $T_{P}$ is the theory of lovely pairs of models of $T$, as defined in [4], then $T_{P}$ has NIP. This provides an interesting class of examples to which Theorem 2.1 applies even when condition (iii) is replaced by (iii)'

The following is Theorem 3.1 from [6].

Theorem 3.4. Suppose $T$ is o-minimal. $T_{P}$ has NIP if, for all $(M, P(M)) \models$ $T_{P}$, the following conditions are satisfied:

(e) for every finite $n \geq 1$, every definable subset of $P(M)^{n}$ is a boolean combination of sets of the form $X \cap Y$ where $X$ is $\emptyset$-definable and $Y$ is L-definable, 
(f) every definable $X \subseteq M$ is definable by a boolean combination of formulas of the form $(\exists \bar{z})[P(\bar{z}) \wedge \varphi(x, \bar{z})]$ where $\varphi(x, \bar{z})$ is an L-formula possibly with parameters,

(g) every open definable $X \subseteq M$ is a finite union of intervals.

The following result establishes that Theorem 2.1 is a generalisation of Theorem 3.4. The proof borrows from the argument used in [6] to prove Theorem 3.4.

Proposition 3.5. Suppose $T$ is o-minimal. The assumptions of Theorem 3.4 imply the assumptions of Theorem 2.1.

Proof. Suppose the assumptions of Theorem 3.4 are satisfied. Let $(M, P(M)) \prec$ $(N, P(N)) \prec(\bar{M}, P(\bar{M})) \models T_{P}$ be such that all three models are sufficiently saturated. Conditions (i) and (iv) are well known consequences of $T$ being o-minimal. Let $b \in \bar{M} \backslash \operatorname{acl}_{L}(N P(\bar{M}))$. By condition (f) and the fact that $(N, P(N)) \prec(\bar{M}, P(\bar{M}))$, to know $t p(b / N)$ it is enough to know which formulas of the form $\psi(y, \bar{a}) \equiv(\exists \bar{z})[P(\bar{z}) \wedge \varphi(y, \bar{a}, \bar{z})]$ belong to $t p(b / N)$, where $\varphi(\bar{y}, \bar{x}, \bar{z})$ is an $L$-formula with no parameters and $\bar{a} \in N^{|\bar{x}|}$. Knowing that $b \notin a c l_{L}(N P(\bar{M}))$, it is enough to consider the case where, for each $\bar{f} \in P(\bar{M})^{|\bar{z}|}, \varphi(y, \bar{a}, \bar{f})$ defines a possibly empty open interval in $\bar{M}$. In this case the set defined by $\psi(y, \bar{a})$ is an open subset of $\bar{M}$. By condition (g) this set is $L$-definable and so, since $(N, P(N)) \prec(\bar{M}, P(\bar{M}))$, it is $L$-definable over $N$. Therefore $t p(b / N)$ is implied by $t p_{L}(b / N)$ in conjunction with the information that $b \notin a c l_{L}(N P(\bar{M}))$. So condition (ii) is satisfied.

Let $n \geq 1$ be finite and $\bar{f} \in P(\bar{M})^{n}$. Let $Z \subseteq P(M)^{n}$ be definable over $N$ such that $\bar{f} \in Z$. By condition (e), $Z$ is a boolean combination of sets of the form $X \cap Y$ where $X$ is $\emptyset$-definable and $Y$ is $L$-definable. Since $(N, P(N)) \prec(\bar{M}, P(\bar{M}))$, we may assume $Y$ is $L$-definable over $N$. It follows that $\operatorname{tp}(\bar{f} / N)$ is implied by $t_{L}(\bar{f} / N)$ in conjunction with $t p(\bar{f} / \emptyset)$. So condition (iii) is satisfied with $\widehat{f}=\bar{f}$.

\section{An example}

We now consider a class of theories studied by Belegradek and Zilber in [1]. Let $\mathbb{R}$ be the real field and $\mathbb{S}$ the unit circle thought of as a subgroup of the multiplicative group of the complex field $\mathbb{C}$. Let $\Gamma(\mathbb{R}) \leq \mathbb{S}$ be a subgroup. Let $\Gamma(\mathbb{R})^{[n]}=\left\{g^{n}: g \in \Gamma(\mathbb{R})\right\}$. Assume $\Gamma(\mathbb{R})$ satisfies the following three conditions:

- $|\Gamma(\mathbb{R})|=\aleph_{0}$,

- $\left|\Gamma(\mathbb{R}) / \Gamma(\mathbb{R})^{[n]}\right|<\aleph_{0}$ for every finite $n \geq 1$,

- for every finite $n \geq 1$, if $X=Y \cap \Gamma(\mathbb{R})^{n}$ for some algebraic set $Y \subseteq \mathbb{C}^{n}$ 
then $X$ is definable over parameters in the group $(\Gamma(\mathbb{R}), \cdot)$ by a positive quantifier-free formula.

Let $L=\{<,+, \cdot, 0,1, \operatorname{Re}(g), \operatorname{Im}(g)\}_{g \in \Gamma(\mathbb{R})}$ where $\operatorname{Re}(g)$ and $\operatorname{Im}(g)$ are suggestively named constant symbols for the real part and the imaginary part of each member of $\Gamma(\mathbb{R})$. Let $T$ be the resulting $L$-theory of $\mathbb{R}$. Let $R e: \mathbb{S} \rightarrow \mathbb{R}$ be the function which assigns to each member of $\mathbb{S}$ its real part. Let $P$ be a new unary predicate and $L_{P}=L \cup\{P\}$. Interpret $P$ such that $P(\mathbb{R})=\operatorname{Re}(\Gamma(\mathbb{R}))$. Let $T_{P}$ be the resulting $L_{P}$-theory of $\mathbb{R}$. Let $\Gamma$ be a new binary predicate and $L_{\Gamma}=L \cup\{\Gamma\}$. Let the suggestively named $\Gamma(\mathbb{R})$ be the interpretation of $\Gamma$ in $\mathbb{R}$. Let $T_{\Gamma}$ be the resulting $L_{\Gamma}$-theory of $\mathbb{R}$. As is noted in $[1], \Gamma(\mathbb{R})=R e^{-1}(\operatorname{Re}(\Gamma(\mathbb{R})))$ and so $T_{P}$ and $T_{\Gamma}$ are definitionally equivalent.

$T_{\Gamma}$ was studied by Belegradek and Zilber in [1]. They gave axioms for it and proved a near model completeness result. Expecting a positive answer, they asked if $T_{\Gamma}$ has NIP. We use Theorem 2.1 to obtain a positive answer.

We check that $T_{P}$ satisfies the assumptions of Theorem 2.1. Let $(M, P(M)) \prec$ $(N, P(N)) \prec(\bar{M}, P(\bar{M})) \models T_{P}$ be such that all three models are sufficiently saturated. Since $T$ is an expansion by constants of the theory of real-closed ordered fields, it is well known that conditions (i) and (iv) are satisfied. We deduce conditions (ii) and (iii) from the results in [1]. The argument overlaps with the reasoning in [1]. Since the predicate $\Gamma$ is $\emptyset$-definable in $T_{P}$ we shall feel free to use it. Re will now denote the real part map from the unit circle in the big model $(\bar{M}, P(\bar{M}))$. So $\Gamma(\bar{M})=R^{-1}(P(\bar{M}))$. Since $L$ contains constant symbols for all real parts and all imaginary parts of members of $\Gamma(\mathbb{R})$, we may assume $\Gamma(\mathbb{R}) \leq \Gamma(M)$. Let $\Gamma(\bar{M})_{d}$ be the largest divisible subgroup of $\Gamma(\bar{M})$. As is observed in $[1], \Gamma(\bar{M})_{d}$ has a direct complement $H$ in $\Gamma(\bar{M})$ such that $|H| \leq 2^{\aleph_{0}}$. Let $\Gamma(\bar{M})_{d}^{T}$ be the torsion subgroup of $\Gamma(\bar{M})_{d}$. Clearly $\left|\Gamma(\bar{M})_{d}^{T}\right| \leq \aleph_{0}$. Let $K=\left\{h \cdot g: h \in H\right.$ and $\left.g \in \Gamma(\bar{M})_{d}^{T}\right\}$.

Given the sufficient saturation of $M$, we may assume $K \leq \Gamma(M)$.

The following is proved in [1] by means of a back-and-forth argument.

Theorem 4.1. Let $F \leq \Gamma(\bar{M})$ be a subgroup with the following properties: ( $\alpha) F=\{k \cdot d: k \in K$ and $d \in D\}$ for some divisible subgroup $D \leq \Gamma(\bar{M})$, (ß) $|F| \leq 2^{\aleph_{0}}$, $(\gamma) \Gamma(\mathbb{R}) \leq F$

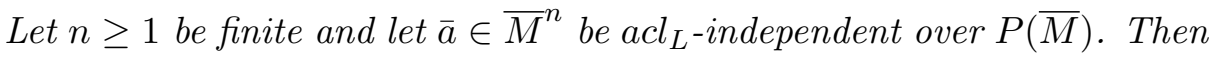
$\operatorname{tp}(F \bar{a})$ is implied by the information so far mentioned in conjunction with $t p_{L}(F \bar{a})$.

Let $b \in \bar{M} \backslash \operatorname{acl}_{L}(N P(\bar{M}))$. Let $F \leq \Gamma(N)$ satisfy conditions $(\alpha),(\beta)$ and $(\gamma)$ and let $\bar{a}$ be a finite tuple from $N$ such that $\bar{a}$ is $a c l_{L}$-independent over 
$P(\bar{M})$. It follows from Theorem 4.1 that $t p(b / F \bar{a})$ is implied by $t p_{L}(b / F \bar{a})$ in conjunction with the information that $b \notin a c l_{L}(N P(\bar{M}))$. It is then clear that $t p(b / N)$ is implied by $t p_{L}(b / N)$ in conjunction with the information that $b \notin a c l_{L}(N P(\bar{M}))$. So condition (ii) is satisfied.

Let $n \geq 1$ be finite and $\bar{f} \in P(\bar{M})^{n}$. Let $F \leq \Gamma(\bar{M})$ satisfy conditions $(\alpha)$, $(\beta)$ and $(\gamma)$ and be such that $\bar{f} \subseteq \operatorname{Re}(F)$. Let $\tilde{F} \leq \Gamma(N)$ satisfy conditions $(\alpha),(\beta)$ and $(\gamma)$ and let $\bar{a}$ be a finite tuple from $N$ such that $\bar{a}$ is $a c l_{L^{-}}$ independent over $P(\bar{M})$. It is clear that the subgroup $\{c \cdot d: c \in F$ and $d \in$ $\tilde{F}\}$ also satisfies conditions $(\alpha),(\beta)$ and $(\gamma)$ and that this is a consequence of $\operatorname{tp}(F / M)$ in conjuction with $\operatorname{tp}(\tilde{F} / M)$. It follows from Theorem 4.1 that $\operatorname{tp}(F / \tilde{F} \bar{a})$ is implied by $t p_{L}(F / \tilde{F} \bar{a})$ in conjunction with $t p(F / M)$. It is then clear that $t p(F / N)$ is implied by $t p_{L}(F / N)$ in conjunction with $t p(F / M)$. Clearly this remains true when $F$ is replaced by any $F^{\prime}=t p(F / M)$. So condition (iii) is satisfied with $\kappa \leq 2^{\aleph_{0}}$ and $\widehat{f}=\operatorname{Re}(F)$.

\section{REFERENCES}

[1] O. Belegradek and B. Zilber. The model theory of the field of reals with a subgroup of the unit circle. J. London Math. Soc., 78:563-579, 2008.

[2] A. Berenstein, A. Dolich and A. Onshuus. The independence property in generalized dense pairs of structures. Preprint 145 on MODNET Preprint server, 2008.

[3] A. Berenstein, C. Ealy and A. Günaydın. Thorn independence in the field of real numbers with a small multiplicative group. Ann. Pure Appl. Logic, 150:1-18, 2007.

[4] A. Berenstein and E. Vassiliev. On lovely pairs of geometric structures. Ann. Pure Appl. Logic, 161:866-878, 2010.

[5] L. van den Dries and A. Günaydın. The fields of real and complex numbers with a small multiplicative group. Proc. London Math. Soc., 93:43-81, 2006.

[6] A. Günaydın and P. Hieronymi. Dependent pairs. Preprint 146 on MODNET Preprint server, 2010.

[7] B. Poizat. A course in model theory. Springer, 2000.

[8] S. Shelah. Stability, the f.c.p., and superstability; model theoretic properties of formulas in first order theory. Ann. Math. Logic, 3:271-362, 1971. 